\title{
LA WEB SEMÁNTICA Y SUS USOS EN LOS PROCESOS DE DOCUMENTACIÓN ARCHIVÍSTICA ORGANIZACIONAL
}

\section{A WEB SEMÂNTICA E SEUS USOS NOS PROCESSOS DE DOCUMENTAÇÃO ARQUIVÍSTICA ORGANIZACIONAL}

\author{
Juan Bernardo Montoya Mogollón * \\ Telma Campanha de Carvalho Madio **
}

\begin{abstract}
Resumen
Introducción: Los procesos de Gestión Documental fueron implementados con el fin de solucionar las diversas dificultades producto de actividades documentales a lo interno de organizaciones. Esas prácticas se complejizaron aún más con la producción y la diseminación de los documentos en soporte digital. Objetivo: El presente artículo tiene como objetivo principal presentar y analizar el modelo de la Web Semántica y sus lenguajes como una contribución para las actividades de gestión de documentos archivísticos digitales que se producen, gestionan y tramitan al interior de las diferentes entidades, principalmente privadas. Metodología: Este trabajo parte de una discusión teórica sobre el tema mencionado, recopilando una amplia literatura que abarca los conceptos de la Gestión Documental alineada con los lenguajes de la Web Semántica para ser aplicados en ambientes empresariales. Resultados: El resultado de esta investigación da cuenta de la elaboración de un proyecto en el que se consiguió aprovechar esas características para la producción, gestión, conservación, preservación y acceso a los usuarios que necesitan cada vez más de una información en tiempos deseados y de forma completa. Conclusiones: La conclusión del trabajo se centra en el reaprovechamiento de las nuevas herramientas tecnológicas que necesitan con urgencia ser aplicadas en áreas como la Archivística y Ciencia de la Información.
\end{abstract}

Palabras clave: Gestión Documental. Web Semántica. Documentos Archivísticos Digitales. Organización Corporativa.

*Doutorando em Ciência da Informação da Universidade Estadual Paulista - Marília. Email: juanmont1981@gmail.com

**Doutora em Ciências da Comunicação pela Universidade de São Paulo. Docente do Departamento de Ciência da Informação da Universidade Estadual Paulista Júlio de Mesquita Filho- Marília. E-mail: telmaccarvalho@marilia.unesp.br

Inf. Inf., Londrina, v. 22, n. 2, p. 437 - 448, maio/ago., 2017. http:www.uel.br/revistas/informacao/ 


\section{INTRODUÇÃO}

La actividad informacional se encuentra día a día en un proceso de desmaterialización permanente sin que la sociedad esté percibiendo que esto está actualmente ocurriendo. Situación suscitada principalmente por el aumento vertiginoso de la innovación tecnológica de la cual se está cada vez más dependiente, por lo que se observa que los individuos están comenzando a establecer relaciones más dinámicas y reciprocas con máquinas computacionales para que estas últimas interpreten las solicitudes de búsquedas informacionales de una manera más eficiente.

Con lo descrito anteriormente, se están desarrollando proyectos que adquieran esa capacidad de establecer comunicaciones de forma más efectiva entre computador-ser humano, como por ejemplo el modelo de la web semántica, el cual pretende construir un entendimiento humano, con el objetivo principalmente de estructurar y recuperar el diverso acervo documental producido en los diferentes dominios.

En lo que se refiere a la organización de la información en diferentes entidades (sean estas públicas o privadas), se ha visto que la actividad funcional en el campo empresarial ha sido configurado de forma compleja. Ante el problema de ausencia de espacio físico para conservación de acervos documentales en papel, se buscó la solución en el proceso de desmaterialización, que consistía en digitalizar y posteriormente concentrar teras y teras de información en los diferentes repositorios. Sin embargo, lo que se observó realmente, fue la transferencia del problema y no su solución, es decir, la dificultad para organizar, conservar y recuperar la información física, cambio solo de soporte.

De esta manera, con el objetivo de realizar una contribución para la gestión documental en soporte digital dentro de ambientes empresariales, el presente trabajo pretende aportar al análisis del desarrollo de la web semántica, como una herramienta de auxilio en los diferentes procesos la actividad documental organizacional.

Inf. Inf., Londrina, v. 22, n. 2, p. 437 - 448, maio/ago., 2017. 


\section{DOCUMENTACIÓN EN PAPEL Y DOCUMENTACIÓN DIGITAL: EL NUEVO DESAFÍO DE LAS ORGANIZACIONES}

La producción documental en soporte físico y electrónico es un activo de vital importancia en cualquier entidad, ya que en ella se plasman las diferentes actividades laborales, la memoria organizacional e institucional y la trazabilidad de cada una de las áreas o departamentos que constituyen la organización en la continuidad del negocio. Por tanto, desde algunas décadas atrás, las empresas han buscado la posibilidad de mejorar las prácticas archivísticas con el objetivo de estructurar el universo informacional a ser conservado y preservado en soporte papel.

¿Y por qué en soporte físico? Principalmente porque gran parte de las empresas nacieron y se fortalecieron durante todo el siglo $\mathrm{XX}$, tiempo en el cual, el documento en papel era - y continúa siendo - el soporte por excelencia, además de la legalidad y de la confianza que brinda, lógicamente con los atributos y obligaciones conocidos hasta el día de hoy. En este sentido, si bien es cierto que la labor del archivista comenzó a tener una relativa importancia en la transición del siglo anterior a este, en el proceso de organizar el acervo documental, el mismo continúa con serias dificultades, principalmente por una cultura organizacional que valoriza la información en su inmediatez, ya que después de cumplir su importancia transaccional activa, muchas veces pasa a ser una molestia y es "transferido" a cuartos que no están en uso o a los rincones de los baños.

Ahora bien, estas dificultades aumentaron aún más con la llegada del documento digital, donde se observó su considerable producción en detrimento del documento tradicional, y desde algunas décadas atrás, la solución del problema que se pensaba resuelto con la digitalización física, se convirtió en un real dolor de cabeza, ya que la forma de conservar la información, solo cambió de soporte: documentos físicos y virtuales conviven actualmente en un caos organizacional, donde los últimos son guardados en costosos repositorios (cloudcomputing) como si fuese una "caja de zapatos" (VAN DIJCK, 2007); además de ser información de toda índole, la cual continúa creciendo de forma exponencial en todas las organizaciones y en la sociedad en general, 
encontrándose distribuida por los diversos sitios fruto de la utilización intensiva del "cloud" (VIDIGAL, 2014, p.1).

Así mismo, esa conducta organizacional de conservar todo tipo de documento que pueda servir de prueba en algún momento determinado, sin establecer un ciclo de vida, ha hecho muchas veces que el conocimiento del archivista sea sobrevalorado, especialmente en la organización, clasificación y conservación adecuada de los documentos que son realmente importantes para la misión organizacional (NORA,1984, p.15).

\section{GESTIÓN DOCUMENTAL: UN ANÁLISIS EN EL ÁMBITO ORGANIZACIONAL}

La gestión documental se creó principalmente como una herramienta de apoyo para que las entidades en general mejoren e estandaricen las actividades documentales, por medio de procesos que establecen la circulación documental desde el momento de su creación hasta su disposición final, pasando por diferentes estados archivísticos, llamados generalmente como archivos de gestión (espacio en el que nace y se gestiona el documento), archivo central (espacio al que pasa un documento después de no ser usado con tanta frecuencia) y archivo histórico o permanente (espacio en el que un documento ha dejado de ser importante para las actividades laborales cotidianas y adquiere una importancia para la memoria y cultura de la empresa).

De la misma manera, existen otro tipo de instrumentos que coadyuvan a que la gestión documental dentro de una organización sean aún más efectiva, de la cual se destacan a saber: las tablas de retención documental, las tablas de valoración documental, los planos de clasificación, los inventarios documentales, el plan institucional de archivo (PINAR), el modelo de requisitos para la gestión de documentos electrónicos, las tablas de control de accesos, entre otros.

Así, se puede argumentar que el avance alcanzado por los procesos de la gestión documental, han ayudado para que se hayan creado políticas internas con el fin de que los documentos estén conservados de una forma

Inf. Inf., Londrina, v. 22, n. 2, p. 437 - 448, maio/ago., 2017. http:www.uel.br/revistas/informacao/ 
idónea, sin embargo, las dificultades aún persisten en este ámbito en pleno siglo XXI, donde se puede analizar gracias a un informe realizado por la compañía Iron Mountain (2014) (empresa que presta servicios de custodia, administración y gerenciamiento de documentos), el riesgo que están enfrentando las compañías con el crecimiento desmesurado del acervo documental en diversos soportes.

\section{WEB SEMÁNTICA}

El desarrollo de la web ha sido un fenómeno sin precedentes. Desde su inicio, cuando Tim Berners-Lee, delineó lo que debería ser el HTML, el protocolo HTTP e la idea de los URL'S con el objetivo de la información de los computadores que llegaban al laboratorio donde este ingeniero trabajaba, lograran conectarse entre sí, "como un sistema de documentación virtual en el cielo" (TED, 2009). El proyecto es conocido actualmente como la World Wide Consortium (W3C) y fue presentado mundialmente en el año de 1990, ofreciendo un importante avance en el campo de la web.

De esta manera, los datos en la web continúa creciendo de forma exponencial y diariamente se conserva una importante cantidad de información, siendo realmente un dolor de cabeza al momento de ser organizada, aún más, al momento de ser recuperada de una forma práctica y entendible.

Se puede constatar esa situación realizando una búsqueda en el motor Google, para verificar que los resultados en la mayoría de las veces es algo desalentador, debido a que este buscador fue diseñado con un sistema de recuperación booleana que impide establecer una comunicación recíproca entre usuario-máquina. "Se escribimos, por ejemplo, la palabra "ketchup" para buscar información sobre un grupo musical, vamos a obtener resultados relativos a restaurantes, recetas, fabricantes, distribuidores e clubes de aficionados de condimentos, e finalmente, lo que queríamos encontrar realmente (posiblemente la dificultad de la recuperación, aumente si el grupo musical fuera poco conocido)" (CASTELLS, 2003, p.3)

El ejemplo citado da cuenta de los vacíos con que hoy en día cuenta internet y la urgencia de construir una herramienta que ayude generar un mejor

Inf. Inf., Londrina, v. 22, n. 2, p. 437 - 448, maio/ago., 2017. http:www.uel.br/revistas/informacao/ 
entendimiento entre las máquinas y los individuos que las utilizan, con el objetivo de que la información "sea clasificada, dotada de estructura e registrada con recurso semánticos explícitos, para que sea procesada por computadores" (CASTELLS, 2003, p. 4.). Esa, entre otras importantes actividades, es la idea de la web semántica.

Ahora bien, el modelo está soportado por una serie de lenguajes o capas que tienen la capacidad de estructurar la información desde una base meramente sintáctica hasta un nivel de complejidad semántica. Es decir, el modelo se encuentra estructurado principalmente por los siguientes lenguajes: URI, Unicode, XML, RDF, OWL, Logic, Proof y Trust, los cuales pueden "codificar" información y convertirla de manera que sea entendible para cualquier usuario.

\section{WEB SEMÁNTICA APLICADA A LA GESTIÓN DOCUMENTAL}

Gracias al importante avance de la web semántica en los últimos años, se ha observado que este modelo ha podido expandirse como herramienta de ayuda en diferentes ámbitos de la ciencia. Se ha visto por ejemplo, interesantes contribuciones en sites de internet, desde donde se puede "comprar un libro, reservar un lugar en un vuelo aéreo, hacer una transferencia bancaria, hasta simular una hipoteca" (CASTELLS, 2003, p.5).

De esta forma, se ha visto la relevancia en la aplicación de estos lenguajes a los procesos documentales que se establecen en ambientes empresariales, en los cuales se tramitan con los documentos desde su propia producción, pasando por la recepción, distribución, trámite, organización, consulta, conservación y disposición final; incluso con documentos digitales, para los cuales se adhirió el proceso de los metadatos para garantizar su autenticidad (ALVES; SANTOS, 2014, p.358).

Precisamente, el concepto de los metadatos ha sido una herramienta que vincula de un modo colaborativo el modelo de la web semántica con el de la gestión documental. Así, en el campo de la archivística, el mismo ha sido definido de manera estándar como "datos estructurados que describen y permiten encontrar, gerencia, comprender y/o preservar documentos 
archivísticos a lo largo del tiempo" (CONSELHO NACIONAL DE ARQUIVOS, 2011; ALVES; SANTOS, 2014, p.362).

En este sentido, la vinculación de los metadatos al proceso de la gestión documental ha contribuido para el tratamiento de la documentación orgánica durante los tres estados del ciclo de vida documental. Los mismos pueden servir para describir contenido e estructura de los documentos de archivo, tanto en los soportes analógicos como en los digitales y por medio de este modelaje, permitir que una agente de software inteligente, pueda descubrir, catalogar y comprender el recurso de una página web (GAMA, 2011, p.57).

Adicionalmente, otra ventaja que se puede observar en los metadatos, es que ellos pueden ser aplicados en diferentes dominios y cada entidad tiene la capacidad de escoger el modelo que mejor se adapte a las necesidades documentales: metadatos aplicados a documentos de forma individual, metadatos aplicados al conjunto o agrupamiento de los documentos, o metadatos aplicados a los sistemas completos de gestión archivística de documentos. La decisión sobre los niveles de aplicación de los metadatos deberán ser especificadas en las políticas y reglas elaboradas por las instituciones para la gestión de metadatos en los sistemas informatizados (INTERNATIONAL ORGANIZATION OF STANDARDIZATION, 2006, 2008; ALVES; SANTOS, 2014, p.364).

Continuando con lo escrito, un lenguaje que puede ayudar a realizar ese proceso de descripción de los metadatos, es el RDF (ResourceDescription Framework), el cual ofrece la posibilidad de describir un recurso y "proporciona una forma homogénea de representar el conocimiento que favorece la interoperabilidad de recursos y aplicaciones (es decir, se maximizan las posibilidades de su reutilización" (CODINA, MARCOS, PEDRAZA, 2009, p.185).

Por lo tanto el lenguaje RDF posee la capacidad de crear una serie de dispositivos semánticos con sintaxis y estructuras de metadatos en un dominio específico, además, también se puede relacionar con otros dominios para que las declaraciones originales mantengan su legibilidad. Esto es posible gracias a la construcción inicial del RDF realizado por el W3C (GAMA 2011, p.57).

Inf. Inf., Londrina, v. 22, n. 2, p. 437 - 448, maio/ago., 2017. 
Con lo citado anteriormente, se puede decir que las ventajas que tiene el uso del lenguaje RDF para las actividades de descripción documentales de las organizaciones son relevantes ya que ofrecen a los usuarios, una mejor estructura de sus documentos y una recuperación basada en un trabajo cooperativo con sistemas computacionales de mayor entendimiento, por medio de una "radical normalización sintáctica y semántica desarrollada por el uso del RDF, lo cual implica que el uso masivo de datos sea accesible a un mayor número de empresas y organizaciones" (SAORÍN, PESET, FERRER, 2013, p.12).

Ahora bien, con la descripción de los recursos en los ambientes empresariales, el proceso es complementado con el uso de lenguajes ontológicos (OWL, Ontology Web Language). Ontología, es un concepto tomado de la filosofía la cual tiene como comprensión, "la explicación sistémica de la existencia y cómo aquella es percibida por los humanos", y aplicada a los sistemas de información, es "considerada como un artefacto del software (o lenguaje formal) diseñado para un conjunto específico de usos y ambientes computacionales (BARCHINI, ÁLVAREZ, HERRERA, 2006, p.5).

La importancia de este lenguaje OWL en el proceso organizacional, se centra en que por medio de este se puede dar una mayor estructura semántica a los procesos informacionales que se generan en el día a día, además, pueden establecer igualmente interoperabilidades documentales entre diferentes sistemas de información, uno de los problemas más recurrentes en el ámbito organizacional.

\section{CONCLUSIONES}

La contribución de la web semántica continúa avanzando vertiginosamente, ofreciendo herramientas a tener en cuenta para la organización del acervo informacional que se localiza en los diferentes y amplios dominios. Sin duda en el campo organizacional, este modelo continuará mejorando los diversos procesos relacionados con la heterogénea información que se genera diariamente fruto de las actividades laborales,

Inf. Inf., Londrina, v. 22, n. 2, p. 437 - 448, maio/ago., 2017. http:www.uel.br/revistas/informacao/ 
estableciendo igualmente que, la responsabilidad de los usuarios es un factor de vital importancia para el uso correcto del modelo, por tanto, es necesario establecer una cultura organizacional desde los cargos de tomas de decisiones para que contribuyan al correcto uso de los procesos documentales.

\section{REFERÊNCIAS}

ALVES, R. C. V. et al. Ciência da Informação, Ciência da Computação e Recuperação da Informação: algumas considerações sobre os métodos e tecnologias da informação utilizados ao longo do tempo. Revista Eletrônica Informação e Cognição, v. 6, n.1, p. 28-40, 2007.

ALVES, R. C. V.; SANTOS, P. C. L. V. A. C.; RODRIGUES, A. C. Metadados arquivísticos: considerações sobre conceitos, tipos e instrumentos. Encontro Nacional de Pesquisa em Ciência da Informação, v. 15, 2014.

ANTONIO, R.; PROENÇA L. A gestão documental na governança da informação. Lisboa: APDSI, 2014. Disponível em:

<http://www.apdsi.pt/uploads/news/id844/Gest\%C3\%A30\%20Documental\%202 014 20141111.pdf>. Acesso em: 03 de agosto de 2015.

ARCHIVO GENERAL DE LA NACIÓN DE COLÔMBIA: Disponível em: <http://www.archivogeneral.gov.co>. Acesso em: 03 de agosto de 2015.

ATHENTO: Smart Document Management: Disponível em: <http://www.athento.com/>. Acesso em: 03 de agosto de 2015.

BARCHINI, G.; ÁLVAREZ, M.; HERRERA, S. Sistemas de Información: nuevos escenarios basados en ontologías. Revista de Gestão da Tecnologia e Sistemas de Informação, v. 3, n.1, 2006. Disponível em: $<$ http://www.scielo.br/pdf/jistm/v3n1/02.pdf >. Acesso em: 03 de agosto de 2015.

CASTELLS, P. La Web Semántica. 2006, 13 p. Disponível em: $<$ http://arantxa.ii.uam.es/ castells/publications/castells-uclm03.pdf>. Acesso em: 03 de agosto de 2015.

CODINA, L. La web semántica: una visión crítica. El Profesional de la Información, v. 12, n.2, mar/abr.,2003. Disponível em:

$<$ http://www.elprofesionaldelainformacion.com/contenidos/2003/marzo/16.pdf03 de agosto de 2015>. Acesso em: 03 de agosto de 2015.

Inf. Inf., Londrina, v. 22, n. 2, p. 437 - 448, maio/ago., 2017. 
CONSELHO NACIONAL DE ARQUIVOS (BRASIL). CÂMARA TÉCNICA DE DOCUMENTOS ELETRÔNICOS. e-ARQ Brasil: modelo de requisitos para sistemas informatizados de gestão arquivística de documentos - Versão 1.1. Rio de Janeiro: Arquivo Nacional, 2011. Disponível em: $<$ http://www.conarq.arquivonacional.gov.br/cgi/cgilua.exe/sys/start.htm>. Acesso em: 23 jun.2011.

FARIA, W. S. A normalização dos instrumentos de gestão arquivística no Brasil: um estudo da influência das Resoluções do Conarq na organização dos arquivos da Justiça Eleitoral Brasileira. 2006. 146 f. Dissertação (Mestrado em Ciência da Informação) - Universidade de Brasília, Brasília, 2006.

FERREIRA, J.; VENTURA, P. O modelo de dados ResourceDescription Framework (RDF) e o seu papel na descrição de recursos. Informação e Sociedade: Estudos, v. 3, n. 2, 2013. Disponível em: $<$ http://www.ies.ufpb.br/ojs/index.php/ies/article/view/15436>. Acesso em: 03 de agosto de 2015.

GAMA, F. A. As contribuições das linguagens de marcação para a gestão da informação da arquivística digital. 2011. 158 f. Dissertação (mestrado) Universidade Estadual Paulista, Faculdade de Filosofia e Ciência, 2011. Disponível em: <http://hdl.handle.net/11449/88196>. Acesso em: 03 de agosto de 2015.

INTERNATIONAL ORGANIZATION OF STANDARDIZATION. Información y documentación - Procesos de gestión de documentos - Metadatos para la gestión de documentos. Parte 1: Principios. ISO 23081-1:2006. Revista Española de Documentación Científica, Madrid, v. 31, n. 2, p. 27-301. 2008. Disponível em: $<$ http://redc.revistas.csic.es/index.php/redc/article/view/429/441>. Acesso em: 10 dez. 2013.

IRON MOUNTAIN Y PWC ÍNDICE DE MADUREZ DEL RIESGO DE LA INFORMACIÓN. Disponível em: <http://www.ironmountain.es/KnowledgeCenter/Reference-Library/View-by-Document-Type/Infographics///IronMountain-and-PWC-Information-Risk-Maturity-Index.aspx>. Acesso em: 03 de agosto de 2015.

JOYANES, L. La computación en nube (cloudcomputing): El nuevo paradigma tecnológico para empresas y organizaciones en la Sociedad del Conocimiento. ICADE. Revista cuatrimestral de las Facultades de Derecho y Ciencias Económicas Empresariales, s/v., n. 76, enero-abril 2009. Disponível em: $<$ http://revistas.upcomillas.es/index.php/revistaicade/article/view/289>. Acesso em: 03 de agosto de 2015.

Inf. Inf., Londrina, v. 22, n. 2, p. 437 - 448, maio/ago., 2017. http:www.uel.br/revistas/informacao/ 
MORALES C. J. M.; HURTADO, G. Integrando las tecnologías de la web semántica en la archivística. Revista de Currículum y Formación de Profesorado. v. 15, n. 1, 2011. Disponível em:

$<$ http://www.ugr.es/ recfpro/rev151ART12res.pdf>. Acesso em: 03 de agosto de 2015.

MOYANO, J. La descripción archivística. De los instrumentos de descripción hacía la web semántica. Anales de Documentación, v. 16, n. 2, 2013. Disponível em: <http://revistas.um.es/analesdoc/article/view/171841>. Acesso em: 03 de agosto de 2015.

NORA, P. Les Lieux de mémoire. Paris: Éditions Gallimard, 1997.vol.1.

RIBEIRO, C. As ciências documentais e a construção da Web Semântica. 2004. Artigo em livro de atas de Conferência Nacional. 17 p. Disponível em: $<$ http://repositorio-aberto.up.pt/handle/10216/67326>. Acesso em: 03 de agosto 2015.

SAORÍN, T. PESET, F. \& FERRER-SAPENA, A. Factores para la adopción de linked data e implantación de la web semántica en bibliotecas, archivos y museos. InformationResearch, v. 18, n.1, 2013.Disponível em: <http://eprints.rclis.org/21005/>. Acesso em: 03 de agosto de 2015.

SILVA, B. A contribuição da web semântica na recuperação da informação arquivística. 2014, 39 f. Trabalho de conclusão de curso (bacharelado Arquivologia) - Universidade Estadual Paulista "Júlio de Mesquita Filho", Faculdade de Filosofia e Ciências.

SILVA M. A; RIBEIRO, F. Das "ciências" documentais à ciência da informação: ensaio epistemológico para um novo modelo curricular. Porto: Edições Afrontamento, 2002.

TED. TECHNOLOGY, ENTERTAINMENT, DESIGN: TED (2009). Tim BernesLee, The next web [Vídeo]. Disponível em: $<$ http://www.ted.com/talks/tim berners lee on the next web?language=en $>$. Acesso em: 03 de agosto de 2015.

TRAMULLAS, J. Tendencias en documentación digital. España: Ediciones Trea, 2006.

VAN DIJCK, J. Mediated memories in the digital age. Stanford University, 2007.

Title

Semantic web and its use in the organizational records processes

Inf. Inf., Londrina, v. 22, n. 2, p. 437 - 448, maio/ago., 2017. http:www.uel.br/revistas/informacao/ 


\begin{abstract}
:
Introduction: The Records Management processes were implemented with the objective of solving the various difficulties resulting from documentary activities within organizations. These practices were further complicated by the production and dissemination of digital records. Objective: The main objective of this article is to present and analyze the Semantic Web model and its languages as a contribution to the management activities of records digital management that are produced, managed and processed, within the different entities, mainly private.Methodology: This work is based on a theoretical discussion on the above mentioned topic, compiling an extensive literature that covers the concepts of Records Management aligned with the languages of the Semantic Web to be applied in business environments. Results: The result of this research reveals the elaboration of a project in which it was possible to take advantage of these characteristics for the production, management, conservation, preservation and access to the users who need more and more information at a desired time and in a complete way.Conclusions: The conclusion of the work is focused on the reuse of the new technological tools that urgently need to be applied in areas such as Archives Science and Information Science.
\end{abstract}

Keywords: Records Management. Semantic Web. Digital Records. Corporation.

\title{
Titulo
}

A web semântica e seus usos nos processos de documentação arquivística organizacional

\section{RESUMO:}

Introdução: Os processos da Gestão Documental foram estabelecidos com o objetivo de dar solução às diversas dificuldades resultado de atividades documentais ao interior das organizações. Práticas essas, que são ainda de maior complexidade pela produção e disseminação de documentos em suporte digital. Objetivo: Portanto, o presente artigo tem como objetivo, apresentar e analisar o modelo da Web Semântica e suas linguagens como contribuição nas atividades de gestão de documentos arquivísticos digitais que são produzidos e disseminados nos diferentes ambientes empresariais.Metodologia: A pesquisa parte de uma discussão teórica sobre o tema mencionado, coletando uma ampla literatura científica que abrange os conceitos da Web Semântica para posteriormente ser aplicados no contexto organizacional. Resultados: O resultado deu conta da elaboração de um arcabouço teórico, em que se conseguiu aproveitar os recursos das duas metodologias apresentadas para a produção, gestão, conservação, preservação e acesso, aos usuários que precisam de informação completa e em curto prazo. Conclusões: A conclusão é focada no reaproveitamento das novas ferramentas tecnológicas que precisam com urgência, ser aplicadas em áreas como a Arquivologia e a Ciência da Informação.

Palavras-chave: Gestão Documental. Web Semântica. Documentos Arquivísticos Digitais. Organização Corporativa.

Recebido: 30.08 .2017

Aceito: 30.09 .2017

Inf. Inf., Londrina, v. 22, n. 2, p. 437 - 448, maio/ago., 2017. 